\title{
Research on Initial Behavior of Screwed Lap Connections of Thin Plates - Experimental Tests and FEM Analysis
}

\author{
Marcin Gryniewicz ${ }^{1 *}$ \\ ${ }^{1}$ Department of Building Structures, Faculty of Civil Engineering and Environmental Sciences, Bialystok University of Technology, \\ Wiejska 45, 15-351 Bialystok, Poland \\ * Corresponding author, e-mail: m.gryniewicz@pb.edu.pl
}

Received: 23 January 2021, Accepted: 22 May 2021, Published online: 10 June 2021

\begin{abstract}
The paper continues the past research on the screwed thin-plate single-lap connections undertaken by the author. This re-search was focused on stressed skin diaphragm action, where it is essential to know values of connections behavior. Until now, initial stiffness is used for common engineering estimations. The main aim of this paper is to compare the experimental results of the shear tests conducted on the connections with the theoretical predictions of their initial behavior obtained from the FEM models. The experiments followed the Eurocode rules for design aided by testing and the European Convention for Constructional Steelwork (ECCS) recommendations. FEM parametric models are introduced as a consideration to use, taking into account screw threads and EPDM washers. In the technical literature, various techniques are described for the modelling of threaded connections. A modification to a known solution is applied in this analysis where a spiral thread is substituted by simple horizontal rings; this simplification allows use of symmetry in a static system, which is particularly desirable for large models such as those prepared in this paper. In contrast to the mentioned known solutions, the thread mod-el presented here has a triangular cross-section related to the geometry used in real screws. The numerical results correspond reasonably well to the test's early stage measurements from the lab experiments and can be useful for initial stiffness examination (e.g. where no data is provided for a particular connector by a producer) or as a base to further research on the model.
\end{abstract}

Keywords

thin-walled structures, screwed connections, FEA, initial shear stiffness

\section{Introduction}

Research on the thin-walled members in a structural design requires consideration of the behavior and strength of its connections. The recent works [1] that have been conducted in the field of "stressed skin diaphragm action" show that the finite element method (FEM) is preferred in practical engineering. Models of the connections are typically difficult to implement because of the numerous effects that are required to be taken into account. An important problem is to determine the degree of complexity of a joint between two structural elements in a static system such that the most adequate behavior is ensured.

A problem needs to be addressed when the contact occurs between different types of structural elements, e.g., when corrugated steel plates are connected with structural bearing elements (e.g., where sheeting acts as a diaphragm). Another issue is the connection between sheets. A common feature of both cases is that the edge joints are discrete and not continuous. This discontinuity is important for the appropriate functioning of a relevant static system. Each joint has to be treated as a chain of connectors, rather than a single continuous connection only. Thus, it is necessary to obtain the single-connector parameters and select a method to implement them in a suitable configuration. To confirm these requirements, experimental investigations of self-drilling screwed connections under shear of thin plates were performed [2] and now, they are verified by a FEM parametric model developed in the ANSYS software. Such models can also be treated as a proposition of alternative tool for manufacturers who want to check their products with reduced costs in relation to lab tests.

For this research, a set of statically loaded single-lap self-drilling screw connections have been tested according to the design code EN 1993-1-3 [3] and ECCS manual no. 124 [4]. Until now, the single value of $0.25 \mathrm{~mm} / \mathrm{kN}$ for 
the slip of connections is applied as initial stiffness to all the screws with overall diameter from $4.1 \mathrm{~mm}$ to $4.8 \mathrm{~mm}$ according to the ECCS Recommendations no. 88 [5]. This value can be compared to the statistical results from the performed tests, and additionally verified by the computational simulations. The simulations are limited to an initial stage of connections work since the linear stiffness is currently recommended to be used for global analyses of buildings. This simplification allowed to perform the complete set of calculations to be compared with laboratory cases.

Some research on screwed and blind bolted connections of thin plates has been presented by Swierczyna and Wuwer [6]. Previously, complex joints in frames composed of cold-formed profiles were investigated (with FEM modeling) by Acharya and Sivakumaran [7]. However, recently, new important works have appeared, simultaneously with this research. Wrzesien et al. [8,9] confirm the need for the proper understanding of connections mechanisms during stressed skin modeling. Another idea, comparable to this presented here, was published by Huynh et al. [10]. They also built a FEM model with assumptions close to those applied here. The differences are discussed later in the paper.

\section{Experimental tests of connections subjected to shear}

\subsection{Tests preparation}

The chosen requirements for the shear tests are introduced in the latest ECCS (European Convention for Constructional Steelwork) recommendations ECCS no. 124 [4]. The recommendations explain all the necessary problems and are consistent with the Eurocode rules concerning design assisted by testing EN 1990 [11]. This research is based on this manual.

First, the properties of the steel samples and fasteners were determined. The basic characteristics of the steel samples (Table 1$)$ are yield stress $\left(0.2 \%\right.$ proof stress, $\left.R_{p 0.2}\right)$, ultimate tensile strength $\left(R_{m}\right)$, and percentage elongation at the fracture $\left(A_{a}\right)$. The steel has been manufactured by a consistent procedure in accordance with the recommendations of the producer and site requirements (e.g., depth setting control, tightening torque, type of tool, and strength and thickness of the substrate). For fasteners (including metal and rubber washers), the nominal dimensions were verified accordingly. The mechanical properties of the fasteners were examined in accordance with the producer's documentation. Fasteners were fixed within $1 \mathrm{~mm}$ of the positions specified on the test samples.
Table 1 Results of the tests series of DX51 steel samples

\begin{tabular}{lccc}
\hline$m_{e}$ & $R_{p 0.2}$ & $R_{m}$ & $A_{a}$ \\
\hline$[\mathrm{GPa}]$ & {$[\mathrm{MPa}]$} & {$[\mathrm{MPa}]$} & {$[\mathrm{MPa}]$} \\
224 & 327 & 392 & 34.1 \\
\hline
\end{tabular}

The five tests per specimen type allowed the variability in the fastening to be established. Fig. 1 presents the arrangement of a test specimen with two fasteners. Tests with a single fastener are also possible. However, in cases where failure modes like curling or distortion of the connection are expected (like in sheared connections of thin plates) a test with two fasteners may provide results with a smaller variation. Thus, it is recommended by the ECCS [4] to conduct tests using two fasteners configuration and then divide an applied load by two to determine a force at a working load in a single fastener.

The rates of loading and deformation were not exceeded $1 \mathrm{kN} / \mathrm{min}$ and $1 \mathrm{~mm} / \mathrm{min}$, respectively. The deformation was measured beyond $3 \mathrm{~mm}$ of the relative displacement. The ultimate resistance of the fastening was taken as the maximum load recorded during the test. It is also recommended to define a failure load in a deformation of $3 \mathrm{~mm}$. A peak load beyond the $3 \mathrm{~mm}$ limit was recorded during the test.

\subsection{Shear resistance and flexibility}

The characteristic resistance of a fastening is derived from a statistical evaluation of the test results (the mean of the test results obtained from a minimum of five tests):
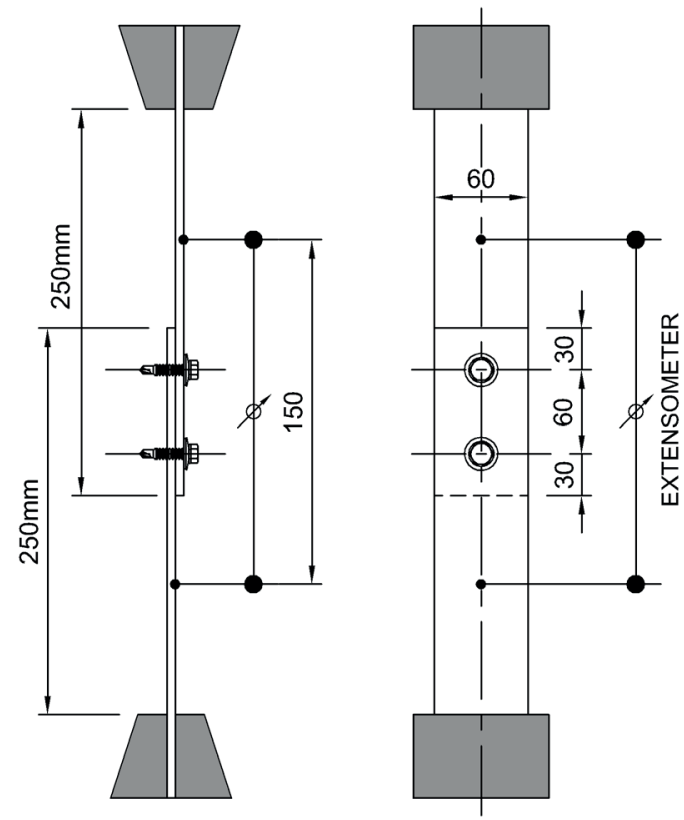

Fig. 1 Shear test specimen with two fasteners 
$R_{k}=R_{m}-k \cdot s_{k}$,

where $k$ is the fractile factor, which can be taken from Annex D of EN 1990 [11] and $s_{k}$ is the standard deviation. The mean of the test results has to be adjusted by taking into account the actual measured resistance of the material in comparison with the nominal resistance. Fractile factor $k$ equals 2.33 for five tests if the coefficient of variation is not available from prior knowledge. The design shear resistance of the connection is defined with the formula given below:

$$
R_{d}=\frac{R_{k}}{\gamma_{M 2}}
$$

where $\gamma_{M 2}$ is the partial factor for the resistance (see corresponding EN 1993-1-3 [3]). Finally, the shear flexibility of a fastening should be determined from:

$c_{h}=\frac{1}{R_{d} / \gamma_{1}} \cdot \frac{\sum a_{h}}{n}$,

where $a_{h}$ is the slip of a fastening at a load equivalent to $R_{d} /$ $\gamma_{1}, \gamma_{1}$ is an appropriate factor, and $n$ is the number of test specimens. For example, for fastening sheets to a substructure in a single fastener test, the appropriate value of $\gamma_{1}$ is 1.5 (which corresponds to the factor used for wind action).

\subsection{Failure modes of connections}

During the tests of thin plates under shear, failure modes like curling or distortion are expected and should be avoided (in continuous connections, e.g., between trapezoidal sheets, such effect should not typically appear). Therefore the tests are performed using two fasteners configuration. It is required to record the failure mode during the tests. Typical modes according to the ECCS manual no. 124 [4] are listed in Table 2 ('applied symbol' in the table is added by the author).

Possible single observations during tests are: I - Elongation of hole, II - Piling of material, III - Necking/Tearing, IV - Pull-out of fastener. The experiment described in this paper has been designed to avoid the joint capacity loss caused by tilting and pull-out. This was achieved by using two screws in a single connection as described above.

\subsection{Experiment details and results}

Plates of different thicknesses (from 'A' to 'G', see Table 3) were used to build a complete and practical set of data. Plates with thicknesses from $0.50 \mathrm{~mm}$ to $1.25 \mathrm{~mm}$ were composed of DX51 steel. One exception was the plate of thickness $1.50 \mathrm{~mm}$ that was fabricated using steel grade S350. This difference was a consequence of the production process established by the supplier. Five specimen assemblies were built for each thickness (a total of 70 plates were necessary to build 35 assemblies). All the specimens were protected by hot-dip galvanizing.

The connectors used in the tests were HILTI company self-drilling screws: 'S-MS' type for plates $0.50 \mathrm{~mm}$ thick and 'S-MD' for the other plates. All the screws had the same diameter of $4.8 \mathrm{~mm}$ and were equipped with steel and sealing washers. They are commonly used by contractors at building sites. These types of connectors were selected based on this practical application. The material for the screws was stainless steel grade 1.4301. Fig. 2 presents the side view of an example of the connected plates. All the experiments have been undertaken using a Zwick/ Roell test machine controlled by a computer unit with dedicated software, testXpert II. The rates of loading and displacement were controlled during the entire loading process as suggested by the manual (rates of $1 \mathrm{kN} / \mathrm{min}$ and $1 \mathrm{~mm} / \mathrm{min}$, respectively were not exceeded). The testing

Table 2 Characteristics of the failure modes of the connections for fasteners under a shear [9]

\begin{tabular}{lcclll}
\hline Applied symbol & Failure mode & I & II & III & IV \\
\hline BF & bearing failure & x & x & & \\
NF & net section failure & x & x & x & \\
SF & shear of fastener & & & & \\
EF & end failure & x & x & x & \\
TF & tilting and pull-out & & & & $x$ \\
\hline
\end{tabular}

Table 3 Statistical results of the shear tests in relation to the slip of a single screw

\begin{tabular}{|c|c|c|c|c|c|c|c|c|}
\hline Symbol & Unit & A & B & $\mathrm{C}$ & $\mathrm{D}$ & $\mathrm{E}$ & $\mathrm{F}$ & G \\
\hline$t$ & {$[\mathrm{~mm}]$} & 0.50 & 0.60 & 0.70 & 0.80 & 1.00 & 1.25 & 1.50 \\
\hline$R_{m}$ & {$[\mathrm{~N}]$} & 1462 & 1519 & 1948 & 2698 & 3490 & 5073 & 5853 \\
\hline$s_{k}$ & {$[\mathrm{~N}]$} & 27.3 & 124.0 & 80.3 & 68.4 & 86.0 & 134.5 & 144.8 \\
\hline$R_{k}$ & {$[\mathrm{~N}]$} & 1398 & 1230 & 1761 & 2539 & 3740 & 4760 & 5516 \\
\hline$c_{h}\left(\gamma_{1}=1.0\right)$ & {$[\mathrm{mm} / \mathrm{kN}]$} & 1.05 & 0.26 & 0.29 & 0.30 & 0.34 & 0.19 & 0.17 \\
\hline$c_{h}\left(\gamma_{1}=1.5\right)$ & {$[\mathrm{mm} / \mathrm{kN}]$} & 0.50 & 0.14 & 0.18 & 0.17 & 0.12 & 0.10 & 0.09 \\
\hline
\end{tabular}


procedure was terminated automatically by the computer system based on the given high thresholds for the force and displacement controls. Tests were performed until the connection was completely broken.

First, the main failure mode of the connection was bearing failure (BF) (see Table 2 and Fig. 3). Shear of the fasteners SF, end failures EF, or tilting and pull-out failures TF did not occur at a characteristic resistance level. The statistical evaluation performed according to the ECCS manual no. 124 [4] and Eurocode EN 1990 [11] is summarized in Table 3.

All the presented results are calculated for a single connector. Fig. 4 shows the example graphs for the complete observation periods of the selected thicknesses to display the range of the performed tests. Each graph consists of five curves for each specimen separately.

\section{FEM parametric analysis}

\subsection{Model data and chosen parameters}

The ANSYS Mechanical software has been used for the FEM modeling of the connections. Its geometry is constructed using a build-in environment called the 'Design Modeller'. The connections configurations are examined according to the complete 3D FEM analysis. It was possible to parameterise all the dimensions to decrease the time of the creation process of every new geometry. The main difference between each sample plate is the thickness of the connected plates. In almost all the cases, the screw type and diameter remain the same. Moreover, all the specimens had the same dimensions in plane. Thus, the main variable is the thickness of a plate $\left(t_{p}\right)$. Other model geometric dimensions are kept constant or depend on the chosen plate thickness (as a function of variable $t_{p}$ ).

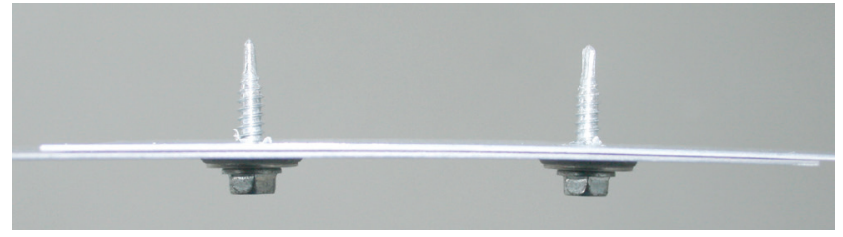

Fig. 2 Example of a connection - side view

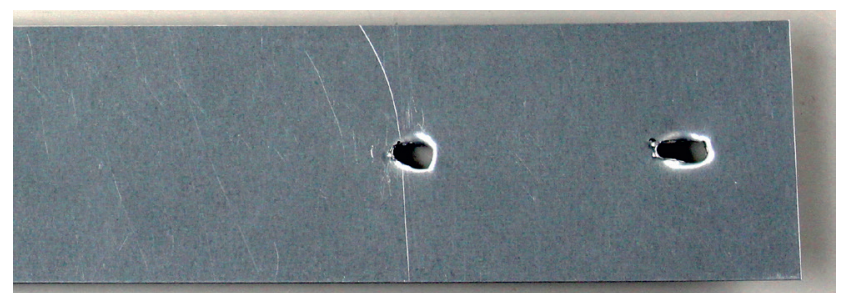

Fig. 3 Example of a connection failure (BF)
The most difficult issue in modeling is the screw-plate contact. Various techniques are available for the modeling of threaded connections with FEM software, such as those described by Oliver [12] or Huynh et al. [10]. A similar solution is applied in this analysis, as described by Oliver [12], where a spiral thread is substituted by simple rings. This simplification allows using symmetry in a static system, which is particularly desirable for large models. In contrast with the cited article, the thread model presented here has a triangular cross-section corresponding to real systems, see Fig. 5. For the thick plates (larger than $1.00 \mathrm{~mm}$ ), two thread rings per plate are applied, and for others, only one ring per plate; this corresponds to the thread spacing of the fastener. Contact type with a 0.2 friction coefficient is set for each surface pair. The main differences to Huynh et al. [10] is that in-plate hole drilling (caused by screw taping) and an EPDM washer are also taken into account here. It is essential to be noted that implicit analysis has been used here (Huynh et al. [10] used the explicit). The decision to use the implicit solver were made since the focus was on the initial behavior of connections under static shear forces.

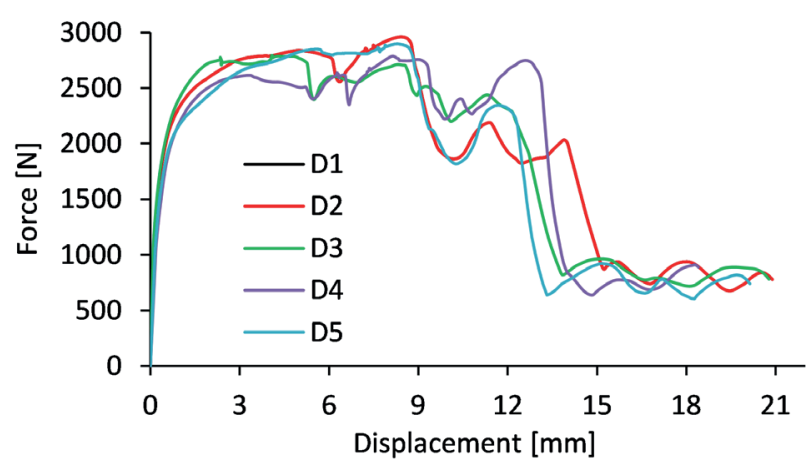

(a) $t=0.80 \mathrm{~mm}$

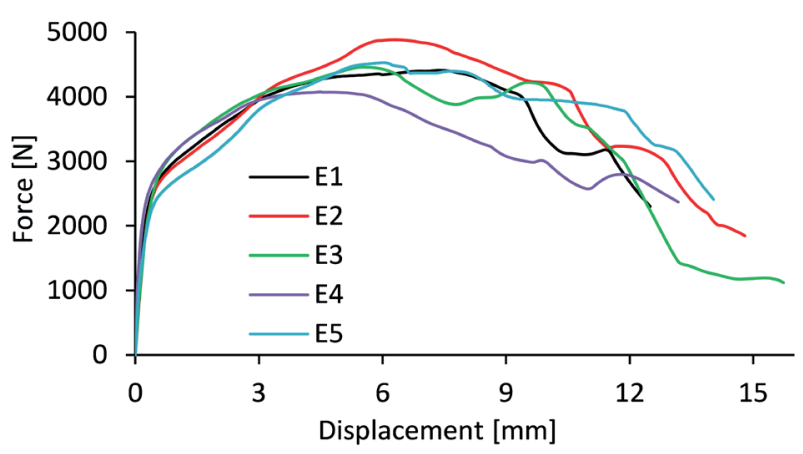

(b) $t=1.00 \mathrm{~mm}$

Fig. 4 Selected example graphs of the complete tests processes for different thicknesses (five tests per curve per thickness) in relation to a single connector 


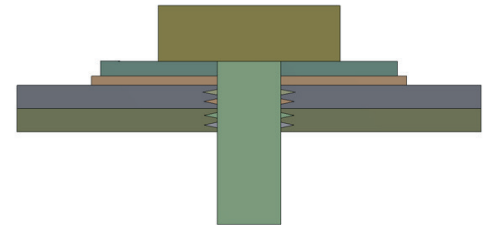

(a)

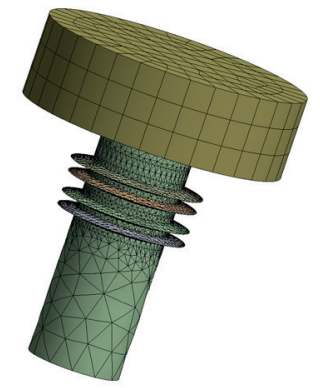

(b)

Fig. 5 Cross-section of a (a) connection model (in the symmetry plane) and (b) 3D view of a screw (for the thickness of plates $t=1.00 \mathrm{~mm}$ )

The plate material model is multi-linear according to the uni-axial tests of the samples done according to ISO 6892-1 [13]. The rubber gasket material is EPDM (ethylene propylene diene monomer) described by the MooneyRivlin two-parameter model, where $C_{10}=123 \mathrm{MPa}$ and $C_{01}=493 \mathrm{MPa}$ [14]. For the screw material, linear characteristics have been chosen. This is a conscious simplification, which enhances the convergence time. All the loads and support conditions correspond to those applied in the experiment. Additionally, a small pretension is applied to the screws to simulate tightening during application of them. Time-stepping with force increment was controlled manually to ensure the best quality of results and solution convergence.

A view of the complete example assembly is shown in Figs. 6 and 7 ('symmetry expansion' feature has been used which means that the symmetric geometry is viewed as a complete model). All parts are modeled using 3D solid finite elements. For the two main plates in a general domain, one layer of hexahedral elements is applied. In the connection area (see the internal ring, Fig. 7) multilayered tetrahedral mesh is used and refined. The interface between these parts is a bonded type. The contact between the plates and the other assembly parts are defined as frictional in the simulations.

\subsection{Results and comparison with experimental tests}

The outputs from the computational analysis of the assembly model are examined qualitatively and quantitatively. First, the types of the deformation of the assemblies have to be confirmed. It can be seen from the deformation images in Fig. 8 that plate curling occurs at the short edges (note that this is the highly scaled deformation). This is the expected behavior, which was also observed in the experiments, even though in this case it clearly appeared at later stages of the application of the force to the specimen. It is essential to avoid effects such as curling during low-force loading to focus on the pure shear only (curling cannot appear in long joints in diaphragms).

Another important effect that also appears in the FEM analysis is screw tilting and pulling-out (Fig. 8). This behavior is a result of using thread solid modeling and 'frictional' contact for the surfaces, corresponding to real connections. Piling of a material with a hole elongation in the numerical model can be seen in Fig. 9. This is a typical effect in connections with fasteners under shear and has also been recorded during the tests. This failure mode predominates during the early stages of the simulations which is the desired behavior, presumably through using two fastener configuration of the connections.

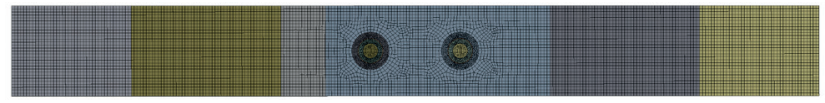

Fig. 6 Top view of the connection

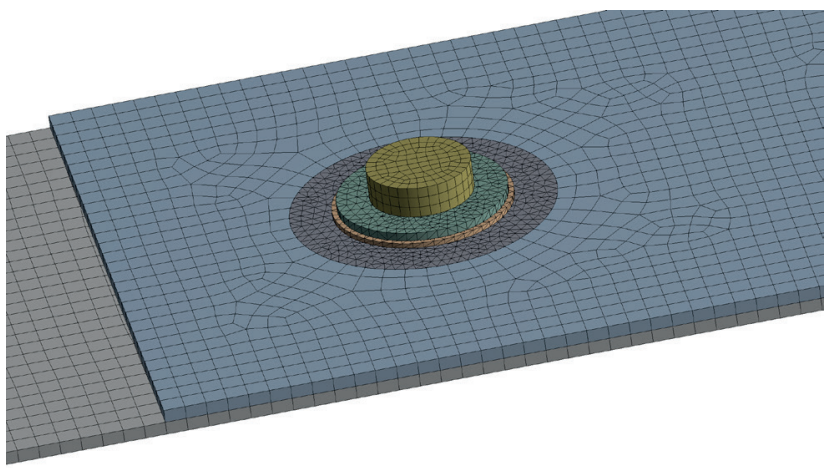

Fig. 7 Connector area of the assembly

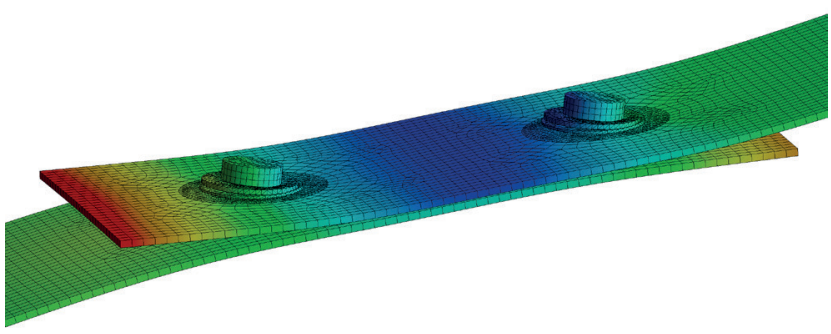

(a) isometric view

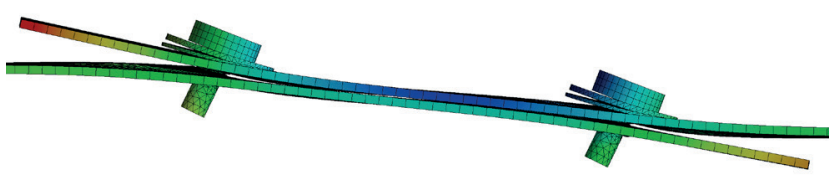

(b) side view

Fig. 8 Scaled deformations of the representative connection 


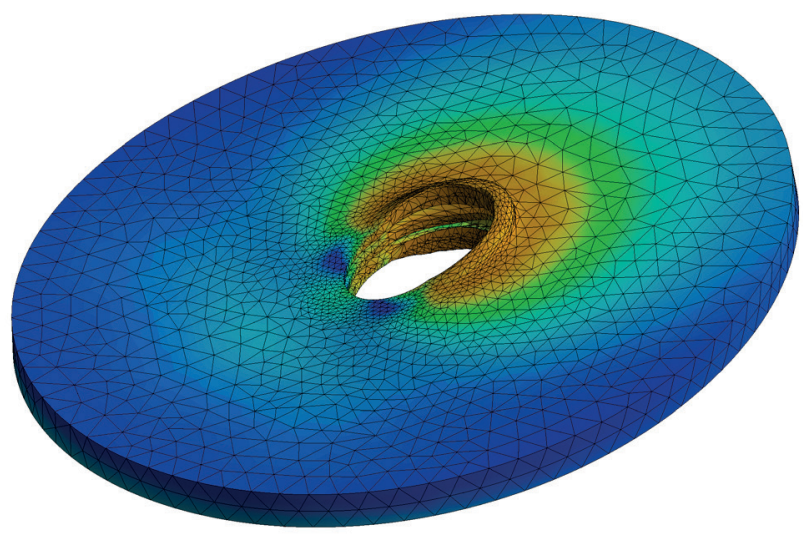

Fig. 9 Scaled deformation with mapped general stress fields around a hole in the internal fragment of a plate

A quantitative analysis of the results is conducted using the charts in Fig. 10. The high nonlinearity of the materials allowed to perform only a part of the complete time experiment simulation. Large plastic deformations are identified in the 'static structural' analysis. An explicit dynamic analysis with the failure of components could be more appropriate in this case. However, such simulations will require a high computing performance and more time when using parametric variables in the systems. Nevertheless, the results obtained from the 'static structural' analysis confirm that it is sufficient and can be useful for research.

Plates of different thickness have been used in the experiment to allow an appropriate variability in the results. The lab tests were checked by the 3D simulations computed in ANSYS Mechanical software. Numerical results correspond reasonably well to measurements from the lab experiments. In this regard, the presented modeling approach shall be considered in further studies, focusing on practical use, e.g., by screws manufacturers. Such a solution would allow saving costs of potential lab tests.

\section{Conclusions}

The complex FEM model of screwed connection has been proposed. This can be the correct approach for calculations of a single connection or a part of a large structure. However, for global static analyses of steel buildings, it is not practical to build every joint in such a way. Those are, e.g., structures with stressed skin diaphragm action involved or frames made of cold-formed sections. An important design problem is to determine the degree of complexity of a joint between two structural elements in a static system such that the most adequate behavior is ensured. For these cases, it is suggested to use more simplified geometric and physical properties of joints [15].
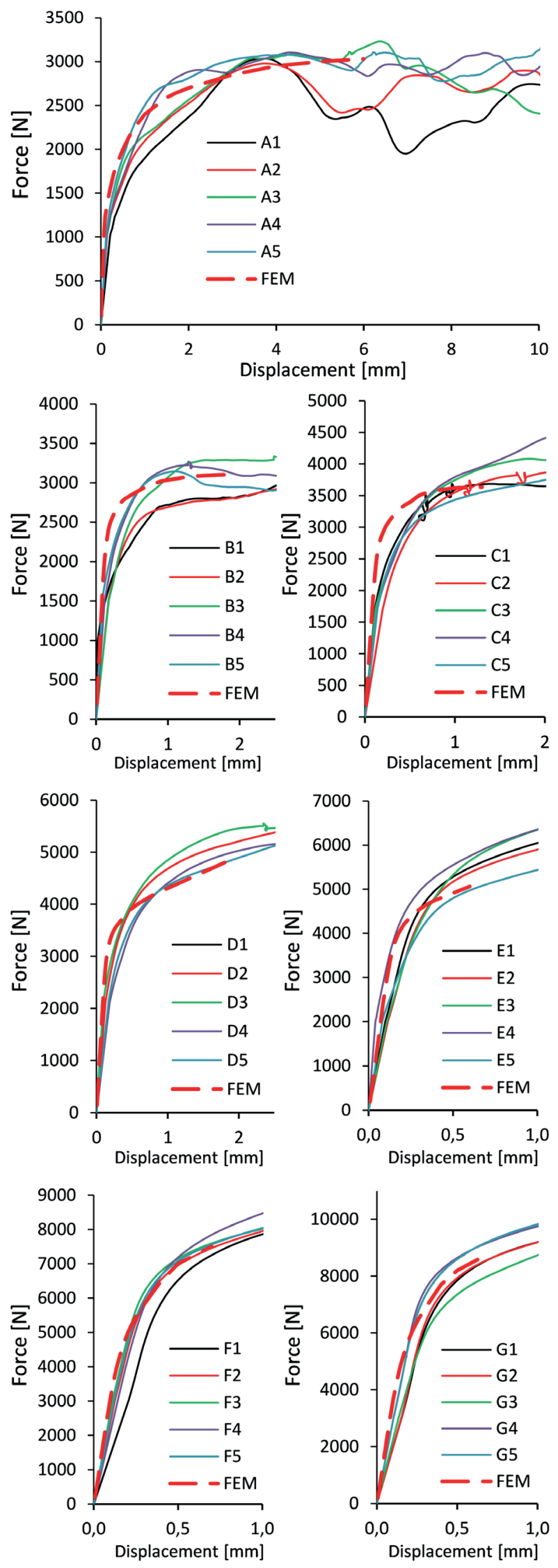

Fig. 10 Comparison of the FEM and experimental results of initial displacements under shear 
For such simplified properties of joints, their initial behavior have to be recognized. FEM models like those presented in this paper can be considered to be used instead of expensive laboratory tests. Obtained results can be useful for the design and research on steel structures with diaphragms. Since static tests are required to be performed on this kind of connections, an implicit analysis appear to be the most relevant. It is well known, that implicit calculations cause difficulties in complex models (notably with contacts), still delivering satisfactory results. It has been proved (e.g., Huynh et al. [10]) that explicit solvers aid with convergence and computation time with acceptable results, but it has to be noted that they are used rather for dynamic and high speed problems. Nevertheless, taking into account a consistency with laboratory static tests and their aim which was the initial stiffness assessment, implicit analysis was worthy to be conducted.

Such analysis is limited to the early stage of connections works which causes that their ultimate failure is unable to be properly assessed. The author's works focus on this problem by improving used methods, e.g., by changing types of finite elements, contacts or solution controls. The current conclusion can be that both types of analyses can be used simultaneously: implicit to determine the initial stiffness and explicit to estimate the failure of connection if necessary. Failure modes identification at the early stage (as performed in this paper) is also necessary which is required to ensure that unwanted failure modes did not occur.

\section{References}

[1] Gryniewicz, M., Szlendak, J. K. "FEM model of the steel building roof includes stressed skin diaphragm action effects", In: Recent Progress in Steel and Composite Structures, Proceedings of the XIII International Conference on Metal Structures, Zielona Góra, Poland, 2016. pp. 93-100.

https://doi.org/10.1201/b21417-12

[2] Gryniewicz, M., Szlendak, J. K. "Experimental tests and model study on self-drilling screws connections subjected to shear", In: 8th European Conference on Steel and Composite Structures EUROSTEEL 2017, Copenhagen, Denmark, 2017, pp. 619-628. https://doi.org/10.1002/cepa.100

[3] BSI "BS-EN 1993-1-3 Eurocode 3: design of steel structures. Part 1-3", British Standards Institution, London, UK, 2010.

[4] ECCS "The Testing of Connections with Mechanical Fasteners in Steel Sheeting and Sections", 2nd eds., ECCS TC7 - TWG 7.10, ECCS-CECM-EKS - European Convention for Constructional Steelwork, Belgium, Brussels, 2009.

[5] ECCS "European Recommendations for the Application of Metal Sheeting Acting as a Diaphragm. Stressed Skin Design. No. 88", Steelwork ECCS-TWG 7.5 European Convention for Constructional, Belgium, Brussels, 1995.
A scatter of the experimental results can be seen in the charts shown in Fig. 10. This effect is also observed in the other author's works [10]. It could be a result of an assembly process, a variability of materials characteristics and parts dimensions (imperfections) or in more detail - e.g., the position of the thread established at the end of the drilling process. These results are compared here to the single lines obtained using FEA, which could be corrected by varying the details of the simulations (e.g., mentioned thread model assumed position). Minimal curling during loading also could affect the stability of the results. All of these aspects are the subject of future work.

\section{Acknowledgments}

The author is grateful for the valuable comments and suggestions to this paper provided by J. Michael Davies and Michael J. Roberts from Structure Cladding Interaction Technology Ltd, University of Manchester.

The paper was prepared at Bialystok University of Technology within a framework of the WZ/WB-IIL/4/2020 project sponsored by Ministry of Science and Higher Education and Higher Education (MNiSW), Poland.

The research was also carried out with the use of software ANSYS 19.2, which is provided to the Bialystok University of Technology on the basis of an agreement between Bialystok University of Technology and ANSYS Inc. (Canonsburg, USA) and MESco Sp. z o.o. (Tarnowskie Góry, Poland).

[6] Swierczyna, S., Wuwer, W. "Evaluation of bearing resistance of blind bolt lap joints", In: 7th European Conference on Steel and Composite Structures EUROSTEEL 2014, Naples, Italy, 2014, pp. 381-382.

[7] Acharya, S. R., Sivakumaran, K. S. "Finite Element Models for Thin-Walled Steel Member Connections", International Scholarly Research Notices, 2012, Article ID 197170, 2012.

https://doi.org/10.5402/2012/197170

[8] Wrzesien, A. M., Lim, J. B. P., MacLeod, I. A., Lawson, R. M. "Stressed skin design of steel sheeting panels - Part 1: shear resistance and flexibility of screw lapped joints", presented at WeiWen Yu International Specialty Conference on Cold-Formed Steel Structures, St. Louis, MI, USA., Nov. 7-8, 2018.

[9] Wrzesien, A. M., Lim, J. B., Macleod, I., Lawson, R. M. "Stressed skin design of steel sheeting panels - Part 2: shear panels with sheeting fixed on all 4 sides", presented at Wei-Wen Yu International Specialty Conference on Cold-Formed Steel Structures, St. Louis, MI, USA., Nov. 7-8, 2018. 
[10] Huynh, M. T., Pham, C. H., Hancock, G. J. "On the Finite Element Modeling of the Screwed Connections of Cold-Formed Steel", In: Proceedings of the 4th Congrès International de Géotechnique Ouvrages -Structures, Singapore, 2018, pp. 224-232. https://doi.org/10.1007/978-981-10-6713-6_21

[11] CEN "EN 1990 Eurocode - Basis of structural design", European Committee for Standardization, Belgium, Brussels, 2010.

[12] Oliver, M. "Modeling threaded bolted joints in ANSYS Workbench", ANSYS Advantage, VI(2), 2012.

[13] PKN "ISO 6892-1 Metallic materials. Tensile testing. Method of test at room temperature", International Organization for Standardization, Geneva, Switzerland, 2016.
[14] Felhős, D., Xu, D., Schlarb, A. K., Váradi, K., Goda, T. "Viscoelastic characterization of an EPDM rubber and finite element simulation of its dry rolling friction", eXPRESS Polymer Letters, 2(3), pp. 157164, 2008. https://doi.org/10.3144/expresspolymlett.2008.21

[15] Gryniewicz, M., Szlendak, J. K. "Research on Self-Drilling Screwed Lap Connections in Steel Diaphragms and the Design Models", System Safety: Human - Technical Facility - Environment, 1(1), pp. 299-306, 2019.

https://doi.org/10.2478/czoto-2019-0039 